\title{
Identifying patients at risk for severe exacerbations of asthma: development and external validation of a multivariable prediction model
}

\author{
Rik J B Loymans, ${ }^{1}$ Persijn J Honkoop, ${ }^{2,3}$ Evelien H Termeer, ${ }^{4}$ Jiska B Snoeck-Stroband, ${ }^{2}$ \\ Willem J J Assendelft, ${ }^{3,4}$ Tjard R J Schermer, ${ }^{4}$ Kian Fan Chung, ${ }^{5}$ Ana R Sousa, ${ }^{6}$ \\ Peter J Sterk, ${ }^{7}$ Helen K Reddel, ${ }^{8}$ Jacob K Sont, ${ }^{2}$ Gerben ter Riet ${ }^{1}$
}

\begin{abstract}
- Additional material is published online only. To view please visit the journal online (http://dx.doi.org/10.1136/ thoraxjn-2015-208138).

For numbered affiliations see end of article.

\section{Correspondence to} Dr Rik Loymans, AMC-UvA, PO Box 22660, Amsterdam 1100 DD, The Netherlands; r.j.loijmans@amc.nl
\end{abstract}

Received 29 November 2015 Revised 9 February 2016 Accepted 26 February 2016

Published Online First 4 April 2016

\section{CrossMark}

To cite: Loymans RJB, Honkoop PJ, Termeer EH et al. Thorax 2016;71:838846 .

\begin{abstract}
Background Preventing exacerbations of asthma is a major goal in current guidelines. We aimed to develop a prediction model enabling practitioners to identify patients at risk of severe exacerbations who could potentially benefit from a change in management. Methods We used data from a 12-month primary care pragmatic trial; candidate predictors were identified from GINA 2014 and selected with a multivariable bootstrapping procedure. Three models were constructed, based on: (1) history, (2) history+spirometry and (3) history+spirometry+Fe $e_{N O}$. Final models were corrected for overoptimism by shrinking the regression coefficients; predictive performance was assessed by the area under the receiver operating characteristic curve (AUROC) and Hosmer-Lemeshow test. Models were externally validated in a data set including patients with severe asthma (Unbiased BIOmarkers in PREDiction of respiratory disease outcomes).
\end{abstract}

Results 80/611 (13.1\%) participants experienced $\geq 1$ severe exacerbation. Five predictors (Asthma Control Questionnaire score, current smoking, chronic sinusitis, previous hospital admission for asthma and $\geq 1$ severe exacerbation in the previous year) were retained in the history model (AUROC 0.77 (95\% Cl 0.75 to 0.80 ); Hosmer-Lemeshow $p$ value 0.35 ). Adding spirometry and $\mathrm{Fe}_{\mathrm{NO}}$ subsequently improved discrimination slightly (AUROC 0.79 (95\% Cl 0.77 to 0.81$)$ and $0.80(95 \% \mathrm{Cl}$ 0.78 to 0.81 ), respectively). External validation yielded AUROCS of 0.72 ( $95 \% \mathrm{Cl} 0.70$ to $0.73 ; 71$ to 0.74 and 0.71 to 0.73 ) for the three models, respectively; calibration was best for the spirometry model.

Conclusions A simple history-based model extended with spirometry identifies patients who are prone to asthma exacerbations. The additional value of $\mathrm{Fe}_{\mathrm{NO}}$ is modest. These models merit an implementation study in clinical practice to assess their utility.

Trial registration number NTR 1756.

\section{INTRODUCTION}

Asthma is a common, chronic inflammatory disease of the airways, which is characterised by airflow limitation and the fluctuating course of its symptoms: wheezing, dyspnoea and cough. ${ }^{1}$ These symptoms are influenced by a wide variety of triggers, such as viral airway infections, exposure to inhaled allergens, cigarette smoke and/or exercise. ${ }^{1}$

\section{Key messages}

What is the key question?

- How can I estimate the risk of an exacerbation the following 12 months in an adult patient with asthma?

What is the bottom line?

- Five simple obtainable history variables added with percentage of predicted FEV1 can predict the occurrence of an exacerbation in the following 12 months; $\mathrm{Fe}_{\mathrm{NO}}$ did not improve predictive performance.

Why read on?

- This is the first prediction model for exacerbations of asthma that can be applied in primary care, has been validated in an external population and is easy to apply in clinical practice.

Occasionally, patients experience sudden flare-ups of symptoms requiring prompt change in treatment (exacerbations), ${ }^{2}$ which result in absenteeism from work or school and additional healthcare utilisation. ${ }^{3}$ Besides controlling symptoms, reducing the risk of exacerbations is a major treatment goal in current asthma guidelines. Some known risk factors for exacerbations, such as current smoking, are modifiable, and stepping up asthma treatment can reduce the risk of exacerbations. ${ }^{4}$ Therefore, identifying patients at high risk for exacerbations is useful $; 5$ in particular, it could guide practitioners to administer appropriate interventions at an early stage to those patients who are likely to benefit. Moreover, according to global initiative for asthma (GINA), ${ }^{1}$ assessment of asthma control should include assessment both of current symptom control and of future risks for adverse outcomes.

Several markers appear to be associated with future asthma exacerbations, for example a history of previous exacerbations ${ }^{6}$ and low $\mathrm{FEV}_{1}$. Composite scores largely based on symptoms such as Asthma Control Questionnaire (ACQ) ${ }^{8}$ and the Asthma Quality of Life Questionnaire predict exacerbations better than single variables. ${ }^{9}$ 
Currently however, multivariable prediction models for exacerbations in primary care asthma population are scarce. Although representing a step in the right direction, some existing models suffer from one or more drawbacks that limit their broad application, such as lacking proper assessment of predictive properties $^{10}$ and development in populations with high medication use and/or exacerbation history. ${ }^{11-13}$ Hence, there is a need for a prediction model to identify patients at risk for exacerbations of asthma, which could be more relevant to clinical practice. The aim of the present study was to construct and validate a prediction model for exacerbations of asthma in adults in a heterogeneous asthma population. For this purpose, we analysed data from a pragmatic trial in primary care, in which exacerbations during 12 months of follow-up were recorded and validated the models in an external data set.

\section{METHODS}

\section{Cohort description}

The model derivation cohort consisted of 611 participants who participated in a pragmatic trial with 12-month follow-up assessing patient preferences and cost-effectiveness of three treatment strategies targeted at achieving different levels of asthma control. Details of the methods and results of the Asthma Control Cost-Utility RAndomized Trial Evaluation (ACCURATE) trial are reported elsewhere. ${ }^{14}$ Eligible patients, recruited from general practices in three regions of the Netherlands, were adults with a doctor's diagnosis of asthma who were prescribed inhaled corticosteroids in any dose or form at least once during the previous year. Exclusion criteria were oral corticosteroid therapy within 1 month before enrolment, significant co-morbidity (at the discretion of the practitioners) and insufficient mastery of the Dutch language. At baseline, online questionnaires on patient asthma characteristics, including the $\mathrm{ACQ},{ }^{8}$ were completed, spirometry was performed, a venous blood sample was collected to determine total $\operatorname{IgE}$ and specific IgE against house dust mite, cat, grass and birch pollens and $\mathrm{Fe}_{\mathrm{NO}}$ was measured (details in online supplementary appendix I). During the study, maintenance asthma medications were adjusted at 3-month intervals, based on 5-item ACQ and spirometry with or without $\mathrm{Fe}_{\mathrm{NO}} \cdot{ }^{14}$ As no significant differences were observed in exacerbations between the three treatment strategies, ${ }^{14}$ we combined the data for the present analysis.

\section{Candidate predictors}

Candidate predictors were identified from the GINA 2014 report, ${ }^{1}$ which lists 17 independent risk factors for exacerbations. We were able to operationalise 14 of these variables from the ACCURATE baseline data set (table 1). We were unable to assess inhaler technique, sputum eosinophilia or blood eosinophilia or pregnancy. Additionally, we evaluated the value of adding $\mathrm{Fe}_{\mathrm{NO}}$ to the model; resulting in 15 predictors assessed.

\section{Outcome}

The dependent variable was: one or more severe exacerbations, based on the American Thoracic Society/European Respiratory Society (ATS/ERS) recommendation ${ }^{15}$ : as a course of oral corticosteroids for at least 3 days, and/or an emergency department visit or a hospitalisation due to increased symptoms of asthma.

\section{Statistical analysis}

Handling of missing data

Eighty-four per cent of the patients had complete data for the candidate predictors; missing values were due to unfinished online questionnaires. In $7 \%$ of patients, information on one variable was missing and $1 \%$ of patients lacked information on $\geq 5$ variables from the 15 candidate predictors. Data on severe exacerbations were nearly complete as prescriptions for systemic corticosteroids were obtained from patients' pharmacies after the study; recall bias for emergency department visits or hospitalisations from the three monthly questionnaires was deemed low. We multiply imputed missing values (generating 10 data

Table 1 Candidate variables for prediction of risk of asthma exacerbation based on Box2-2B of the GINA 2014 report and their operationalisation* for the predictor analysis

\begin{tabular}{|c|c|c|}
\hline & Risk factor for exacerbations (GINA 2014) & Operationalisation in ACCURATE database \\
\hline 1 & Uncontrolled asthma symptoms & Asthma Control Questionnaire (ACQ-5; continuous) \\
\hline 2 & High SABA use & $\geq 1$ puff a day during the last 7 days before baseline \\
\hline 3 & Inadequate ICS: not prescribed & No ICS prescribed at baseline \\
\hline \multirow[t]{2}{*}{4} & Inadequate ICS: poor adherence & Medication Adherence Rating Scale (continuous) \\
\hline & Inadequate ICS: incorrect inhalation technique & Not available \\
\hline 5 & Low FEV1 & Prebronchodilator FEV1\% predicted (continuous) \\
\hline 6 & Major psychological problems & Self-reported use of antidepressants \\
\hline 7 & Major socioeconomic problems & Income (ordinal) \\
\hline 8 & Exposures: smoking & Current smoking \\
\hline 9 & Exposures: allergen exposure if sensitised & $\begin{array}{l}\text { Positive-specific IgE titres to house dust mite and/or grass and/or birch pollenst } \\
\text { and/or IgE positivity to cat or dog combined with ownership }\end{array}$ \\
\hline 10 & Co-morbidities: obesity & Body mass index (continuous) \\
\hline 11 & Co-morbidities: rhinosinusitis & Self-reported complaints of chronic sinusitis \\
\hline \multirow[t]{3}{*}{12} & Co-morbidities: confirmed food allergy & Self-reported food allergy \\
\hline & Pregnancy & Not available \\
\hline & Sputum/blood eosinophilia & Not available \\
\hline 13 & Ever intubated or intensive care & Self-reported hospitalisation (ever) for asthma \\
\hline 14 & $\geq 1$ severe exacerbation in last 12 months & Self-reported steroid burst/hospital admission for asthma in the previous year \\
\hline
\end{tabular}


sets) by iterative chained equations using all variables to replace missing values. ${ }^{16}$

\section{Development of the model}

Fourteen candidate predictors identified from GINA (table 1) plus $\mathrm{Fe}_{\mathrm{NO}}$ were assessed during the construction of our models. Continuous variables were analysed as such. Predictors were selected using bootstrapped backward selection by multivariable fractional polynomial modelling. ${ }^{17}$ Variables were selected if the mean bootstrap inclusion fraction (BIF) exceeded $66.7 \%$ out of 1000 bootstrapped replications across the 10 imputed data set.

Three models were constructed a priori, based on: (1) history, (2) history+spirometry and (3) history+spirometry+Fe $\mathrm{FO}_{\mathrm{NO}}$, using logistic regression analysis. For the history-only model, only those candidate predictors were assessed that are usually available for practitioners from history-taking and/or the medical record (items 1-4 and 5-14, table 1). The coefficients of the predictors were corrected for overoptimistic predictions by multiplying them by a fixed heuristic shrinkage factor ${ }^{18}$ (see details in online supplementary appendix I). To facilitate clinical application of the model, we developed a risk score, based on the regression coefficients. ${ }^{19}$

\section{Performance of the model}

The predictive performance of the prediction model was assessed for discrimination, by calculating the area under the receiver operating characteristic curve (AUROC) and for calibration (goodness-of-fit), by drawing calibration plots and the Hosmer-Lemeshow (HL) test. ${ }^{20}$ In addition, we calculated the 10th, 50th and 90th centiles of the distribution of predicted probabilities. The effect of addition of spirometry and subsequently $\mathrm{Fe}_{\mathrm{NO}}$ to the history model was assessed by net reclassification improvement $(\mathrm{NRI})^{20}$ (see details in online supplementary appendix I). The three models were validated externally in the Unbiased BIOmarkers in PREDiction of respiratory disease outcomes (U-BIOPRED) data set $(n=504)^{21}$ (details in online supplementary appendix I). All statistical analyses were carried out in Stata/SE V.13.1 (Stata, College Station, Texas, USA).

\section{RESULTS}

\section{Cohort}

Data of 611 patients were analysed. Baseline patient characteristics (table 2) demonstrated a population with, on average, normal lung function (mean prebronchodilator FEV1 91.3\% predicted; SD 15.4) and a low level of symptoms (mean ACQ-5 score 1.01; SD 0.95). However, the large SDs and wide ranges observed in baseline characteristics reflected a suitably heterogeneous asthma population. Eighty patients (13.1\%) experienced one or more severe exacerbations during 12 months follow-up; a minimum of five events per variable modelled was therefore available. ${ }^{22}$ Marked differences were seen at baseline between patients with and without exacerbations in use of long-acting $\beta_{2}$-agonists, overuse of short-acting $\beta_{2}$-agonists, previous asthma hospitalisation and having experienced an exacerbation in the previous year. All correlations between candidate predictors were below 0.7 , reducing the chance of collinearity.

\section{Prediction models}

The variables selected in the first model (history only) were: ACQ-5 score, current smoking, chronic sinusitis, previous hospitalisation for asthma and treatment with a course of oral corticosteroids in the previous year (table 3; see online supplementary appendix II table A1). The AUROC was 0.77 (95\% CI 0.75 to 0.80 ; figure $1 \mathrm{~A})$. Predicted and actual risks were in agreement for the history-based model (figure 2, panel

Table 2 Baseline characteristics of the model derivation population*; bold variables are candidate predictors

\begin{tabular}{|c|c|c|c|c|}
\hline & $\begin{array}{l}\text { Patients without } \\
\text { exacerbation }(n=531)\end{array}$ & $\begin{array}{l}\text { Patients with } \\
\text { exacerbation }(n=80)\end{array}$ & Total $(n=611)$ & $\begin{array}{l}\text { Observations } \\
\mathrm{n}(\%)\end{array}$ \\
\hline Sex $\%$ female & 67.0 & 77.5 & 68.4 & $611(100)$ \\
\hline Mean age years (SD; range) & $39.0(9.2 ; 17-55)$ & $42.1(8.4 ; 18-51)$ & $39.4(9.1 ; 17-55)$ & $611(100)$ \\
\hline Income (mode range in Euros) & $>50.000$ & $35.000-50.000$ & $>50.000$ & $592(96.9)$ \\
\hline Body mass index $\mathrm{kg} / \mathrm{m}^{2}$ (SD; range) & $26.3(5.3 ; 13.0-56.8)$ & $26.8(5.7 ; 17.4-41.9)$ & $26.4(5.4 ; 13.0-56.8)$ & $590(96.6)$ \\
\hline Asthma duration years (SD; range) & $18.0(13.1 ; 0-51)$ & $23.0(13.4 ; 0-46)$ & $18.6(13.2 ; 0-51)$ & $569(93.1)$ \\
\hline Current smokers \% & 12.2 & 26.6 & 14.1 & $605(99.0)$ \\
\hline Previous smokers \% & 31.9 & 40.4 & 32.9 & $591(96.7)$ \\
\hline Chronic sinusitis \% & 9.2 & 23.4 & 11.0 & $599(98.0)$ \\
\hline Atopy $\%$ total $\lg E>100 \mathrm{kU} / \mathrm{mL}$ & 50.5 & 51.4 & 50.6 & $569(93.1)$ \\
\hline Self-reported food allergy \% & 14.3 & 15.8 & 14.5 & $593(97.1)$ \\
\hline Exposure to sensitised allergens & 71.1 & 67.0 & 71.2 & $569(93.1)$ \\
\hline Self-reported antidepressants use \% & 5.7 & 8.8 & 6.1 & $611(100)$ \\
\hline ACQ-5 score (SD; range) & $0.93(0.86 ; 0.0-4.8)$ & $1.57(1.25 ; 0.0-5.6)$ & $1.01(0.95 ; 0.0-5.6)$ & $593(97.1)$ \\
\hline Using inhaled corticosteroids \% & 80.9 & 89.7 & 82.1 & $592(96.9)$ \\
\hline Beclomethasone equivalent dose (SD; range) & $773(619 ; 0-4000)$ & $1216(794 ; 0-4000)$ & $832(662 ; 0-4000)$ & $592(96.9)$ \\
\hline LABA use $\%$ & 46.9 & 64.1 & 49.2 & $592(96.9)$ \\
\hline High $S A B A$ use $\geq 1$ puff/day, $\%$ & 26.8 & 47,4 & 29.5 & $593(97.1)$ \\
\hline Medication Adherence Rating Scale score (SD; range) & $3.53(0.55 ; 1.7-5)$ & $3.58(0.62 ; 1.6-4.6)$ & $3.53(0.56 ; 1.6-5)$ & $593(97.1)$ \\
\hline Ever hospitalised for asthma \% & 10.3 & 21.6 & 11.7 & $588(96.2)$ \\
\hline Exacerbation previous year $\% \dagger$ & 7.7 & 39.7 & 11.8 & $600(98.2)$ \\
\hline FEV1\% predicted (SD; range) & $92.1(15.1 ; 36.8-147.0)$ & $85.5(16.1 ; 46.0-120.0)$ & $91.3(15.4 ; 36.8-147)$ & $588(96.2)$ \\
\hline $\mathrm{Fe}_{\mathrm{No}} \mathrm{ppb}(\mathrm{SD} ;$ range) & $25(23 ; 5-228)$ & $32(48 ; 5-297)$ & $26(28 ; 5-297)$ & $585(95.7)$ \\
\hline
\end{tabular}


Table 3 ORs and coefficients* of logistic prediction models for severe asthma exacerbations

\begin{tabular}{|c|c|c|c|c|c|c|}
\hline & \multicolumn{2}{|l|}{ History } & \multicolumn{2}{|l|}{ History+spirometry } & \multicolumn{2}{|c|}{ History+spirometry+Fe ${ }_{\mathrm{NO}}$} \\
\hline & $\mathrm{OR}(95 \% \mathrm{Cl})$ & Coefficient & OR $(95 \% \mathrm{Cl})$ & Coefficient & OR $(95 \% \mathrm{Cl})$ & Coefficient \\
\hline ACQ (per 0.5 score) & 1.55 (1.22 to 1.98$)$ & 0.208 & 1.52 (1.19 to 1.94$)$ & 0.198 & 1.52 (1.19 to 1.94$)$ & 0.195 \\
\hline Current smoking & $2.13(1.08$ to 4.17$)$ & 0.712 & $1.83(0.93$ to 3.62$)$ & 0.570 & $1.77(0.89$ to 3.50$)$ & 0.532 \\
\hline Chronic sinusitis & 2.09 (1.02 to 4.28$)$ & 0.694 & 2.49 (1.17 to 5.31$)$ & 0.860 & 2.39 (1.11 to 5.14$)$ & 0.813 \\
\hline Ever admitted asthma & 2.28 (1.16 to 4.49$)$ & 0.778 & 1.90 (0.95 to 3.82$)$ & 0.606 & $1.88(0.94$ to 3.79$)$ & 0.590 \\
\hline Oral steroids previous year & 3.52 (1.68 to 7.36$)$ & 1.188 & $3.69(1.74$ to 7.80$)$ & 1.229 & 3.76 (1.79 to 7.94$)$ & 1.236 \\
\hline FEV1\% predicted (per 10\%) & & & $0.97(0.95$ to 0.99$)$ & -0.281 & 0.97 (0.95 to 0.99$)$ & -0.270 \\
\hline $\mathrm{Fe}_{\mathrm{NO}}($ per $10 \mathrm{ppb})$ & & & & & 1.01 (1.00 to 1.01$)$ & 0.059 \\
\hline Intercept & & -4.595 & & -0.491 & & -0.737 \\
\hline
\end{tabular}

1A), with the HL test yielding no indication of a poor fit ( $\mathrm{p}$ value 0.35 ).

In the second model, addition of spirometry to the history model (table 3) increased the AUROC to 0.79 (95\% CI 0.77 to 0.81 ) (figure $1 \mathrm{~A}$ ); calibration improved slightly (figure 2, panel $2 \mathrm{~A})$, HL test $\mathrm{p}$ value 0.54 .

The third model, with $\mathrm{Fe}_{\mathrm{NO}}$ added to the second model (table 3) changed the AUROC to 0.80 (95\% CI 0.78 to 0.81 ; figure 1A) with a similar calibration (figure 2, panel $3 \mathrm{~A}$ ), $\mathrm{HL}$ test $\mathrm{p}$ value 0.44 . The 10 th, 50th (median) and 90th centiles of the distribution of the predicted probabilities are shown in the online supplementary appendix table A2, figure A1 appendix II). NRI tended to favour addition of spirometry to the history model (total NRI 0.094 for the patients with exacerbations with cut-off $50 \%$; when adding $\mathrm{Fe}_{\mathrm{NO}}$ to the model with spirometry, there was little reclassification (see online supplementary table A3 appendix II)). To improve applicability we checked in the first (history only) model whether ACQ-5 and ACQ-6 (ACQ-5 plus short-acting $\beta_{2}$-agonist use frequency) could be exchanged. Similarly, we checked the exchangeability of prebronchodilator and postbronchodilator FEV1\% predicted in the second model (with spirometry added). Predictive properties of the models remained virtually identical with the exchanged predictors. In the score system for both the history and history+spirometry models (table 4), higher scores indicated increased risk for a severe exacerbation over the next 12 months (figure 3; see online supplementary table A4 appendix II).

\section{External validation}

U-BIOPRED participants had poor asthma symptom control and low lung function; $55.6 \%$ had an asthma exacerbation in the previous year (see online supplementary table A5 appendix II). The exacerbation risk was higher than in the derivation data set, with $34.9 \%$ experiencing a severe exacerbation during 1 year follow-up. Discriminative properties of the models were lower in the external validation set (AUROC $0.72 \quad(95 \%$ CI 0.70 to 0.73 ), 0.72 (95\% CI 0.71 to 0.74 ) and 0.72 (95\% CI 0.71 to 0.73 )) for the three models, respectively (figure $1 \mathrm{~B}$ ). The calibration of the history model demonstrated evident underestimation (circles above the line) in the first seven deciles (relatively low risk groups). Nevertheless, in the models with history + spirometry and history + spirometry $+\mathrm{Fe}_{\mathrm{NO}}$ added, the calibration in lower-risk deciles was better, but in the upper four deciles (predicted probabilities $>60 \%$ ), predicted probabilities
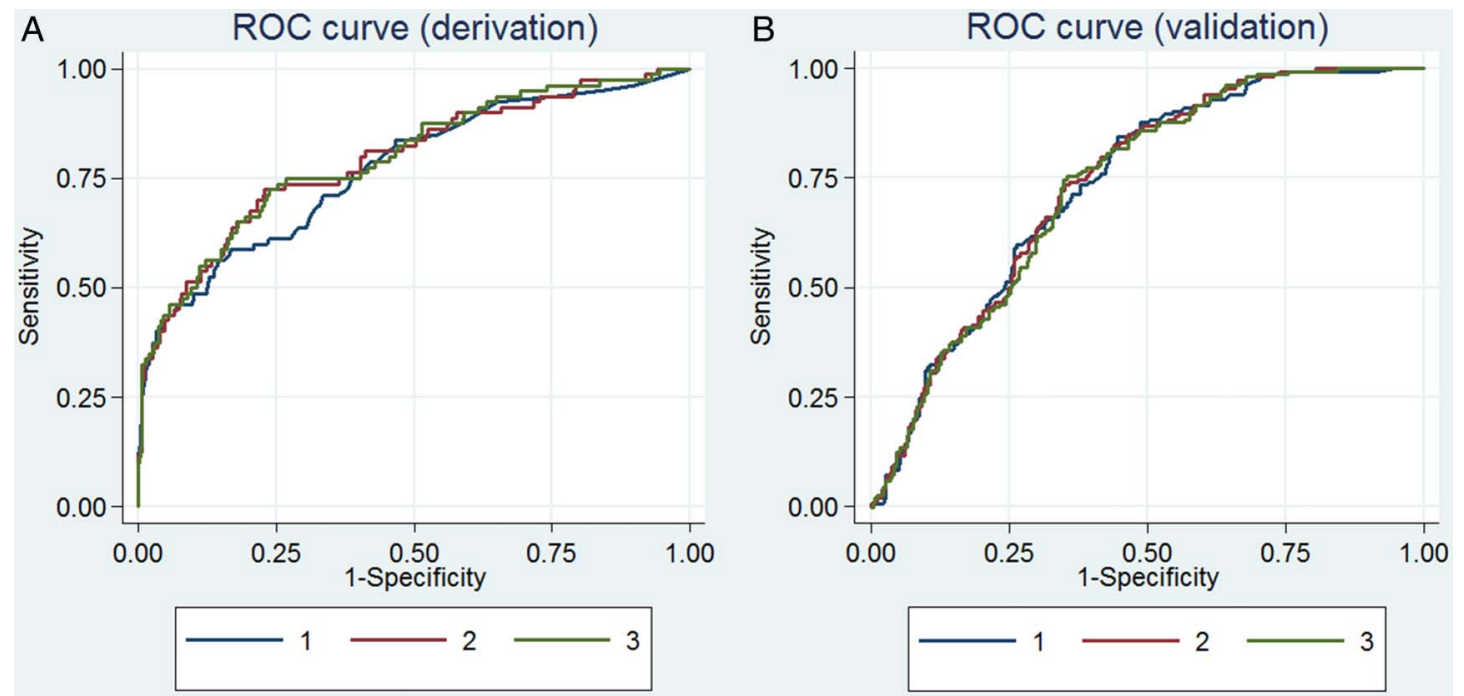

Figure 1 Receiver operating characteristic (ROC) discrimination curve for severe asthma exacerbation prediction models based on history (1), history+spirometry (2) and history+spirometry+FeNO (3); on panel A for the derivation (Asthma Control Cost-Utility RAndomized Trial Evaluation) cohort and on panel B for the validation (Unbiased BIOmarkers in PREDiction of respiratory disease outcomes) cohort. 

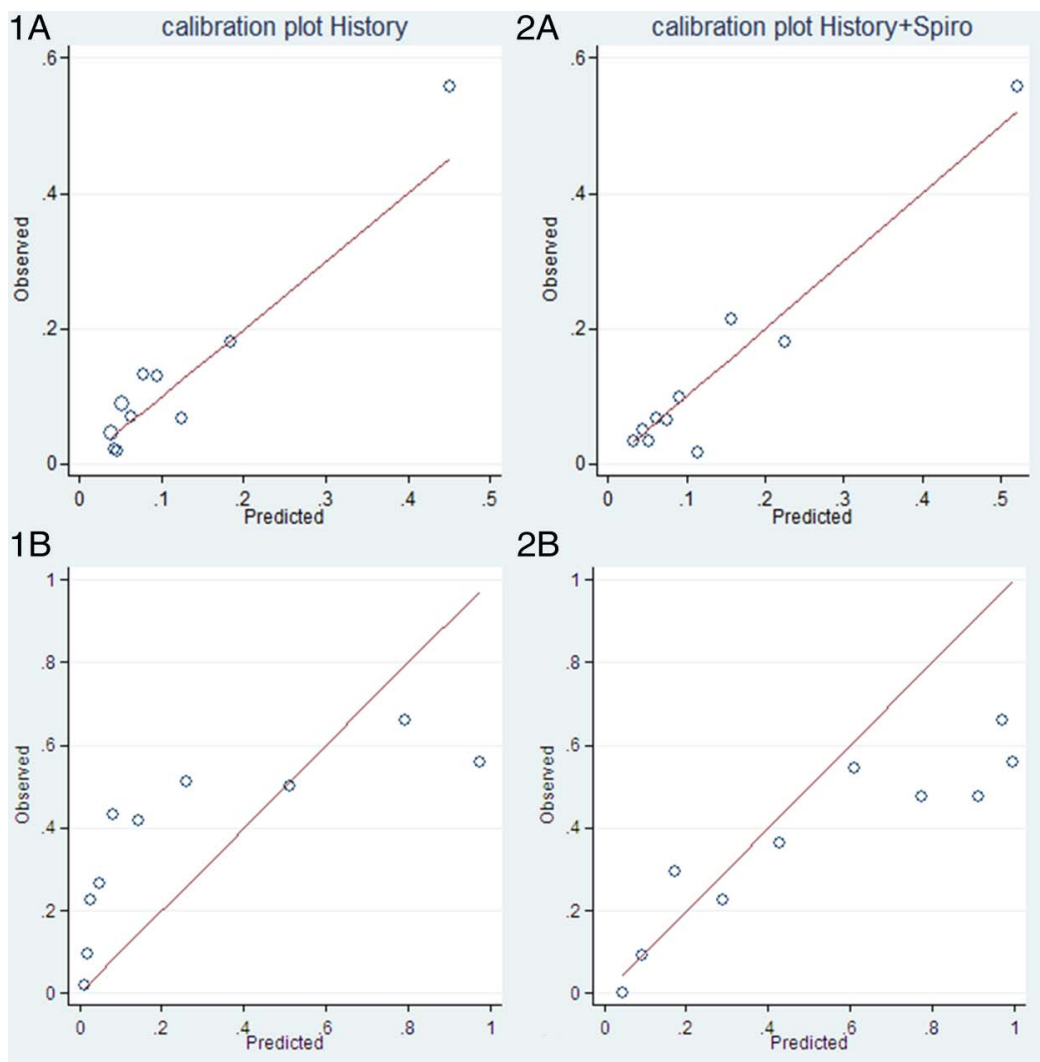

2B

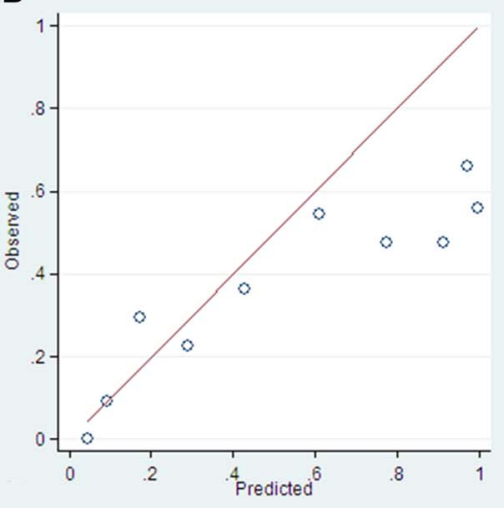

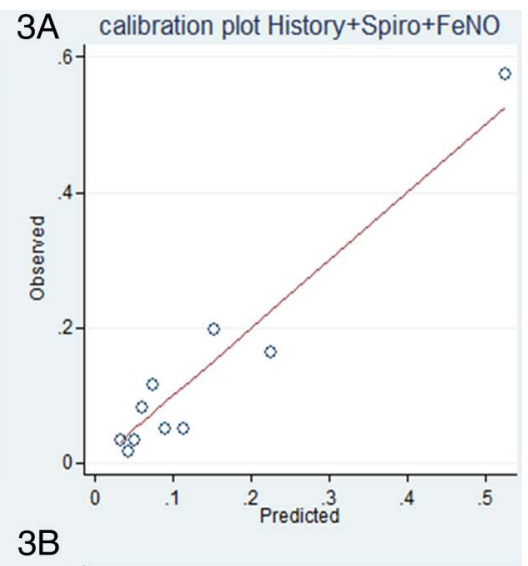

3B

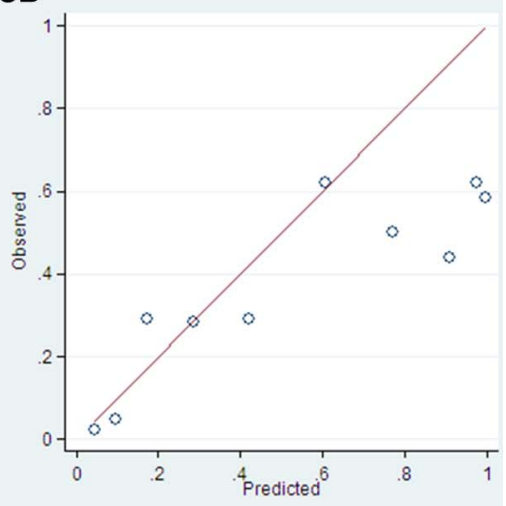

Figure 2 Calibration plots for models based on history (1A), history+spirometry (2A) and history+spirometry+Fe $\mathrm{F}_{\mathrm{NO}}(3 \mathrm{~A})$ in the derivation set shown in the upper three panels. Calibration plots in the validation data set are shown in the lower three panels (1B, $2 B$ and $3 B$, respectively). On the $x$-axis the probability as predicted by the model, on the $y$-axis the probabilities as observed in the data. Circles represent mean predicted probabilities in 10 deciles; the closer the circles are to the straight line, the closer the predicted probabilities from the model are in agreement with the observed probability in the data set.

were higher than observed risk (HL test for all models $<0.05$; figure 2, panels 1B-3B).

\section{DISCUSSION}

The aim of this study was to develop a clinically relevant prediction model to calculate the probability that a patient with

Table 4 Construction of the asthma risk score for the history and history+spirometry model.

\begin{tabular}{llll}
\hline & & Points \\
\cline { 3 - 4 } Factor & & History & History+spirometry \\
\hline ACQ score & $<0.75$ & 0 & 0 \\
& $0.75-1.50$ & 1 & 1 \\
& $>1.5$ & 4 & 4 \\
Current smoking & No & 0 & 0 \\
& Yes & 3 & 2.5 \\
Chronic sinusitis & No & 0 & 0 \\
& Yes & 3 & 3.5 \\
Ever admitted for & No & 0 & 0 \\
asthma? & Yes & 3 & 2.5 \\
Steroids previous year? & No & 0 & 0 \\
& Yes & 5 & 5 \\
Spirometry & $>90$ & & 0 \\
(\% predicted) & $80-90$ & & 0.25 \\
& $<80$ & & 0.50 \\
Total score (range) & & $0-18$ & $0-18$ \\
\hline ACQ, Asthma Control Questionnaire. & &
\end{tabular}

asthma will experience a severe exacerbation during the following year. A model with five easily accessible variables (ACQ-5 score, self-reported current smoking, self-reported chronic sinusitis, previous admission for asthma and a severe exacerbation in the previous year) added with prebronchodilator $\mathrm{FEV}_{1} \%$ predicted performed well on external validation. The addition of $\mathrm{Fe}_{\mathrm{NO}}$ to this model did not improve predictive performance.

This study confirms and extends previous studies ${ }^{6}{ }^{11}$ showing that a previous exacerbation is among the strongest predictor for future exacerbations. With the exception of self-reported chronic sinusitis, the other predictors in our model also belong among the variables most frequently reported to be associated with risk of exacerbations. Each of the predictors in our model (except chronic sinusitis) was also identified in previously published models, such as the TENOR risk score (in severe asthma or difficult-to-treat asthma), ${ }^{12}$ a risk score for exacerbations (from a clinical trial database in patients with $\geq 1$ exacerbations in the previous year and suboptimal symptom control on treatment), ${ }^{13}$ the severity of asthma score, ${ }^{11}$ validated in patients with moderate to severe persistent asthma and the profile of asthma risk, ${ }^{10}$ derived from a health maintenance organisation cohort with patients who had been hospitalised for asthma the previous 2 years or had received at least two dispensings of antiasthma medication in the year before recruitment. Measures of asthma control were included in all these four models whereas previous hospitalisations, recent exacerbations and spirometry measures occurred in three out of four models. Chronic sinusitis is associated with severe asthma, ${ }^{23}$ but it has rarely been assessed as a predictor of exacerbations. The mechanism, if any, is still 

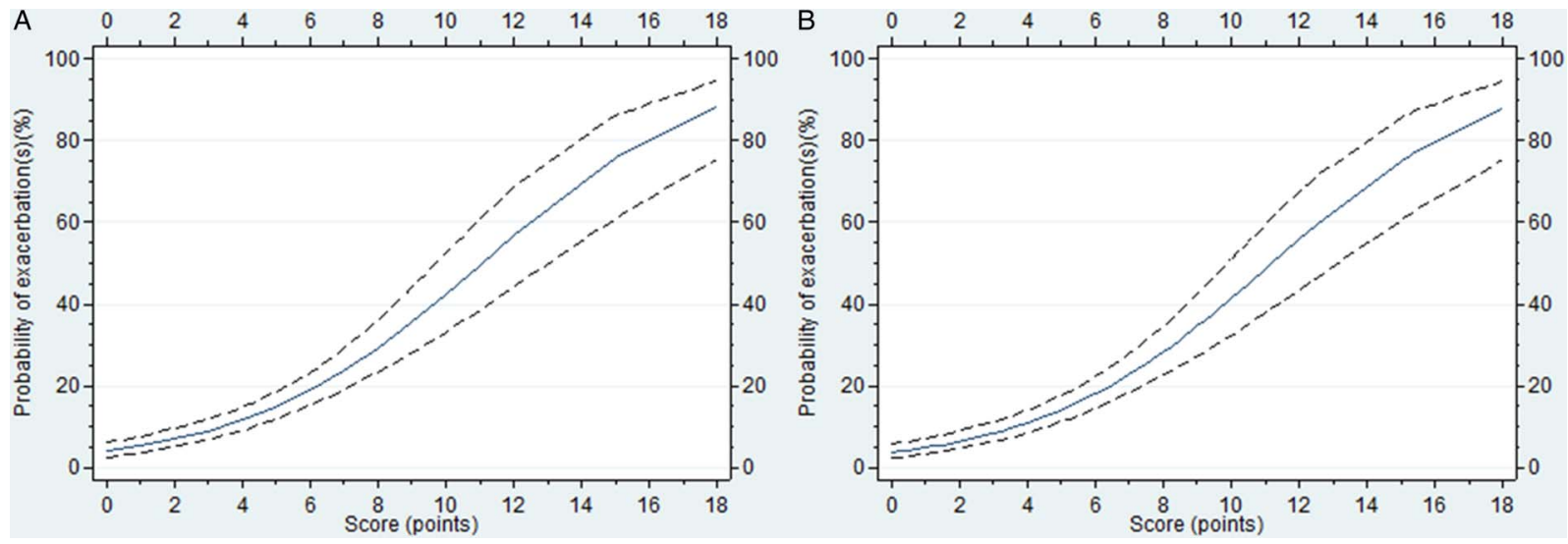

Figure 3 Predicted probabilities of at least one exacerbation during the next 12 months (y-axis) versus the points accredited by the score system (x-axis) for the history model (1) and history+spirometry model (2).

unknown although allergic triggers or nasal polyps may be involved.

Although ethnicity, age, gender and education level have been reported as predictors of exacerbations, ${ }^{9} 1012$ it is unlikely that demographic factors are as strong a predictor as clinical factors. They were also not listed as risk factors for poor asthma outcomes by GINA ${ }^{1}$; they were therefore not assessed in our study.

Our study has several strengths: the ACCURATE trial had a pragmatic design, with few exclusion criteria. Patients were recruited in primary care and treatment by a pulmonary specialist was not an exclusion criterion. This population therefore reflects a broad, relatively unselected asthma population with considerable variability in the baseline patient characteristics, facilitating the development of a stable prediction model. The high level of completeness of data in a large $(n>600)$ cohort, predictor selection by bootstrapping and application of shrinkage probably contributed to the preservation of good model performance on external validation in a different population with more severe asthma and a higher exacerbation risk.

The use of the standardised definition of the outcome variable (severe exacerbations by ATS/ERS criteria) ${ }^{15}$ increases the interpretation ${ }^{24}$ and applicability of this model in clinical practice. The score system enables immediate application of the model in daily practice without using any resources.

Nevertheless, we acknowledge the following limitations. Since our model was developed using data from a randomised trial, treatment effects may have affected the predictions in two ways: either treatment could have been a predictor itself, or treatment could have interacted with other predictors. The first possibility was checked in an additional analysis. We found that treatment was not selected as a predictor when offered in addition to the history-based model (mean BIF 36.8). The latter possibility is less plausible: the treatment algorithms were targeted at different levels of asthma symptom control, based on ACQ score. However, the candidate predictors we assessed were reported by GINA as predictors for exacerbations independent of symptoms. Nevertheless, the repetitive application of each treatment algorithm could have reduced the predictive value particularly of ACQ and $\mathrm{Fe}_{\mathrm{NO}}$ by preventing events happening (possibly introducing bias). Using repeated measurements of ACQ and $\mathrm{Fe}_{\mathrm{NO}}$ in the model may have improved its predictive capacity. We chose not to do so because the collection of repeated measurements complicates clinical application. A second limitation may be the limited number of exacerbations: some authors recommend a minimum of 10 events per predictor, however this criterion may be too conservative. ${ }^{22}$ It also hampered any testing of interactions between predictors with sufficient statistical power. The third limitation is that our definition of an exacerbation was not identical to the definition as proposed by the ATS/ERS ${ }^{15}$ : we could not verify whether patients visiting an emergency department and those hospitalised were treated with systemic corticosteroids. Given the Dutch healthcare system, in which the general practitioners act as gatekeepers to secondary care, the protocol was based on the presumption that all patients visiting the emergency department and those hospitalised were treated with systemic corticosteroids. Finally, an important limitation is that the history model's calibration was lower on external validation in the U-BIOPRED data set, with exacerbation risk underestimated for many patients. Adding spirometry and $\mathrm{Fe}_{\mathrm{NO}}$ values, however, improved the agreement of observed and predicted probabilities, except for some miscalibration overestimation when predicted risks exceeded $60 \%$. Clinical consequences of this potential overestimation of risk are probably limited: most practitioners presumably will consider changing interventions at risks $<60 \%$.

To increase clinical applicability, and assess the added value of objective parameters separately, we built three models, based on: (1) history, (2) history + spirometry and (3) history + spirometry $+\mathrm{Fe}_{\mathrm{NO}}$. The history-only model, which does not require any equipment, performed well on internal validation. However, on external validation, while discrimination remained high, calibration decreased substantially. In particular, predicted risks up to $25 \%$ were too low, which might lead to undertreatment if the model were applied to clinical practice. It should be noted, however, that this miscalibration was observed in a population quite different from the population the model was derived in: the U-BIOPRED population largely consists of patients with severe asthma and this is reflected in an annual exacerbation risk of about $35 \%$ against $13 \%$ in the ACCURATE cohort. Additional validation studies in primary care populations may clarify the history model's performance in other primary care cohorts containing the necessary predictors and severe exacerbations as the outcome.

Adding spirometry to the history model resulted in a modest increase in predictive value and, more importantly, in considerable improvement of calibration in the external validation sample. The remaining miscalibration pertained to predicted risks of $60 \%$ and higher. However, these overestimations may not be important, as in clinical practice most physicians are likely to feel comfortable with intervening once the risk of 
severe exacerbation exceeds $50 \%$, limiting potential overtreatment. Although the use of spirometry presupposes equipment, training and time, it is currently considered to be part of standard asthma care, ${ }^{1}$ so FEV1 values should be used additionally as a predictor when available. Moreover, since the last major revision of the GINA report in 2014, after diagnosing asthma, spirometry is recommended to be used primarily for risk assessment rather than for therapy adjustment. ${ }^{25}$

We also considered $\mathrm{Fe}_{\mathrm{NO}}$ as an additional predictor and as a surrogate for sputum or blood eosinophilia (which were not collected in this trial) even though the association is not strong. ${ }^{26}$ The present data suggest that there is no added value of $\mathrm{Fe}_{\mathrm{NO}}$ on top of history and spirometry to identify patients at risk for future severe exacerbations.

Both additions were assessed by NRI. Reclassification, using arbitrary prediction cut-offs for preventive action of $25 \%$ and $50 \%$, also tended to favour adding spirometry values to the history model.

According to the GINA strategy report, ${ }^{1}$ asthma control means not only good symptom control, but also control of future risk; both of these should be assessed periodically. The added value of the present risk score is that an individual risk for an exacerbation for the next 12 months can be calculated in a simple way. By combining information on the most important risk factors (as listed by GINA) practitioners gain information on future risks in a more explicit way: exacerbation risk in percentage (with 95\% CI) rather than the mere presence or absence of risk factors in a particular patient. Moreover, it prevents practitioners from targeting solely at a single risk factor. When targeting at for example recent exacerbations, a practitioner omits other relevant information. Notably, a model using solely recent exacerbations to predict future exacerbations yielded an AUROC of 0.66.

Although there are no criteria for the level of exacerbation risk that should prompt practitioners to take action, the score system shown in table 4, online supplementary table A3 and figure A2 (appendix II) may assist practitioners in making decisions based on the patients' individual risk. If a patient with good symptom control (ACQ<0.75) during a scheduled visit scores above a particular cut-off (eg, 6 points, corresponding to a predicted risk of nearly $20 \%$ of a severe exacerbation in the next 12 months), the practitioner may consider implementing risk reduction strategies such as providing a written action plan, assessing inhaler technique and adherence, increasing the frequency of review, removal of modifiable risk factors or increasing the treatment step. Conversely, given that many practitioners are not amenable to decreasing treatment steps, ${ }^{27} 28$ our prediction model may also support such decreases, for example by a dose reduction when a patient with stable and well controlled asthma scores $<3$ points (corresponding to a risk of exacerbation of $<9 \%$ ). After additional validation in different (first, second and third line care) clinical settings, studies in a day-to-day practice should point us how this score can assist practitioners in an optimal way (eg, as an app or automatically derived scoring systems from the electronic patient record). The merits of this scoring system may be dependent on the healthcare system it is used in.

\section{CONCLUSION}

In a suitably heterogeneous primary care asthma population with a $13 \%$ chance of experiencing a severe exacerbation within the next year, we found that a simple prediction model containing five medical history items and spirometry identified patients at risk for an exacerbation in the coming year (AUROC 0.79).
Predictive properties remained largely intact on external validation (AUROC 0.72) in a population with more severe asthma and a higher $(35 \%)$ exacerbation risk. If validation in a suitable primary care data set yields similar findings, these models would merit an implementation study in clinical practice to assess their utility.

\section{Author affiliations}

${ }^{1}$ Department of General Practice, Academic Medical Center-University of Amsterdam (AMC), Amsterdam, The Netherlands

${ }^{2}$ LUMC Quality of Care Institute, Leiden University Medical Center (LUMC), Leiden, The Netherlands

${ }^{3}$ Department of Public Health and Primary Care, Leiden University Medical Center (LUMC), Leiden, The Netherlands

${ }^{4}$ Department of Primary and Community Care, Radboud University Nijmegen Medical Centre (RUNMC), Nijmegen, The Netherlands

${ }^{5}$ Experimental Airway Disease, National Heart and Lung Institute, Imperial College London \& Royal Brompton NIHR Biomedical Research Unit, London, UK

${ }^{6}$ Respiratory Therapeutic Unit, GlaxoSmithKline, Stockley Park, UK

${ }^{7}$ Department of Respiratory Medicine, Academic Medical Center-University of Amsterdam (AMC), Amsterdam, The Netherlands

${ }^{8}$ Clinical Management Group, Woolcock Institute of Medical Research, University of Sydney, Sydney, Australia

Correction notice This article has been corrected since it was published Online First. Data in table 4 has been updated.

Acknowledgements We kindly thank the members of the U-BIOPRED Study Group for sharing their data for the external validation. The members of the U-BIOPRED Study Group are: Kamran Tariq, NIHR Southampton Respiratory Biomedical Research Unit, Clinical and Experimental Sciences, NIHR-Wellcome Trust Clinical Research Facility, Faculty of Medicine, University of Southampton, UK; Ratko Djukanovic, NIHR Southampton Respiratory Biomedical Research Unit, Clinical and Experimental Sciences and Human Development and Health, Southampton, UK; Peter Howarth, NIHR Southampton Respiratory Biomedical Research Unit, Clinical and Experimental Sciences and Human Development and Health, Southampton, UK; Graham Roberts, NIHR Southampton Respiratory Biomedical Research Unit, Clinical and Experimental Sciences and Human Development and Health, Southampton, UK; Patrick Dennison, NIHR Southampton Respiratory Biomedical Research Unit, Clinical and Experimental Sciences, NIHR-Wellcome Trust Clinical Research Facility, Faculty of Medicine, University of Southampton, UK; Annelie F. Behndig, Umeå University, Sweden; Wim van Aalderen, Academic Medical Centre, University of Amsterdam, The Netherlands; Rene Lutter, Academic Medical Centre, University of Amsterdam, The Netherlands; Ariane Wagener, Academic Medical Centre, University of Amsterdam, The Netherlands; Elisabeth Bel, Academic Medical Centre, University of Amsterdam, The Netherlands; Kees van Drunen, Academic Medical Centre, University of Amsterdam, The Netherlands; Pieter-Paul Hekking, Academic Medical Centre, University of Amsterdam, The Netherlands; Paul Brinkman, Academic Medical Centre, University of Amsterdam, The Netherlands; Koos Zwinderman, Academic Medical Centre, University of Amsterdam, The Netherlands; Simone Hashimoto, Academic Medical Centre, University of Amsterdam, The Netherlands; Peter J. Sterk, Academic Medical Centre, University of Amsterdam, The Netherlands; Paolo Montuschi, Università Cattolica del Sacro Cuore, Italy; Nadia Mores, Università Cattolica del Sacro Cuore, Italy; Giuseppe Santini, Università Cattolica del Sacro Cuore, Italy; Salvatore Valente, Università Cattolica del Sacro Cuore, Italy; Christos Rossios, Imperial College, UK; Kian F. Chung, National Heart and Lung Institute, Imperial College, London, UK; David Gibeon, Imperial College, UK; Uruj Hoda, Imperial College, UK; João Pedro Carvalho da Purificação Rocha, Royal Brompton and Harefield NHS Foundation Trust, UK; Adesimbo Sogbesan, Royal Brompton and Harefield NHS Foundation Trust, UK; lan Adcock, National Heart and Lung Institute, Imperial College, London, UK; Ioannis Pandis, Data Science Institute, Imperial College, London, UK; Julaiha Gent, Royal Brompton and Harefield NHS Foundation Trust, UK; Andrew Menzies-Gow, Royal Brompton and Harefield NHS Foundation Trust, UK; Louis J. Fleming, National Heart and Lung Institute, Imperial College, London, UK; David Gibeon, National Heart and Lung Institute, Imperial College, UK; Yi-ke Guo, Data Science Institute, Imperial College, UK; Stephen J. Fowler, Respiratory and Allergy Research Group, University of Manchester, UK; Ashley Woodcock, Respiratory and Allergy Research Group, University of Manchester, UK; Alan Knox, Respiratory Research Unit, University of Nottingham, UK; Dominick E. Shaw, Respiratory Research Unit, University of Nottingham, UK; Davide Campagna, Department of Clinical and Experimental Medicine, University of Catania, Italy; Rosalia Emma, Department of Clinical and Experimental Medicine, University of Catania, Italy; Charles Auffray, European Institute for Systems Biology and Medicine, CNRS-ENS-UCBL-INSERM, CIRI-UMR5308, Lyon, France; Betrand De Meulder, European Institute for Systems Biology and Medicine, 
CNRS-ENS-UCBL-INSERM, CIRI-UMR5308, Lyon, France; Diane Lefaudeux, European Institute for Systems Biology and Medicine, CNRS-ENS-UCBL-INSERM, CIRI-UMR5308, Lyon, France; Alexander Mazein, European Institute for Systems Biology and Medicine, CNRS-ENS-UCBL-INSERM, CIRI-UMR5308, Lyon, France; Hassan Ahmed, European Institute for Systems Biology and Medicine, CNRS-ENS-UCBL-INSERM, CIRI-UMR5308, Lyon, France; Amphun Chaiboonchoe, European Institute for Systems Biology and Medicine, CNRS-ENS-UCBL-INSERM, CIRI-UMR5308, Lyon, France; Norbert Krug, Fraunhofer Institute for Toxicology and Experimental Medicine, Hannover, Germany; Jens Hohlfeld, Fraunhofer Institute for Toxicology and Experimental Medicine, Hannover, Germany; Klaus Bonnelykke, Faculty of Health and Medical Sciences, University of Copenhagen, Denmark; Hans Bisgaard, Faculty of Health and Medical Sciences, University of Copenhagen, Denmark; Jens Hohlfeld, Fraunhofer ITEM; Philipp Badorrek, Fraunhofer ITEM; Cornelia Faulenbach, Fraunhofer ITEM; Anna James, Karolinska Institutet, Sweden; Ann-Sofie Lantz, Karolinska University Hospital \& Karolinska Institutet, Sweden; Sven-Erik Dahlen, Centre of Allergy Research, Karolinska Institutet, Stockholm, Sweden; Barbro Dahlen, Sven-Erik Dahlen, Centre of Allergy Research, Karolinska Institutet, Stockholm, Sweden; Elisabeth Henriksson, Karolinska Institutet, Sweden; Nikos Lazarinis, Centre of Allergy Research, Karolinska Institutet, Stockholm, Sweden; Karin Strandberg, Lung and Allergy Clinic, Karolinska Institute, Stockholm, Sweden; Craig Wheelock, Centre of Allergy Research, Karolinska Institutet, Stockholm, Sweden; Gunilla Hedlin, Dept. Women's and Children's Health and Center For Allergy Research, Karolinska Insitute, Stockholm, Sweden; Roelinde Middelveld, Karolinska Institutet, Sweden; Karin Strandberg, Karolinska University Hospital \& Karolinska Institutet, Sweden; Jacek Musial, Dept. of Medicine, Jagiellonian University Medical College, Krakow, Poland; Ildiko Horvath, Semmelweis University, Budapest, Hungary; Gabriella Galffy, Semmelweis University, Budapest, Hungary; M. Szentkereszty, Semmelweis University, Budapest, Hungary; Katherine M. Smith, University of Nottingham, UK; Lilla Tamasi, Semmelweis University, Budapest, Hungary; Massimo Caruso, Dept. Clinical and Experimental Medicine, University of Catania, Italy; Pascal Chanez, Department of Respiratory Medicine, Immunology Lab, Aix Marseille University, France; Laurie Pahus, Department of Respiratory Medicine, Immunology Lab, Aix Marseille University, France; Urs Frey, University Children's Hospital, Basel, Switzerland; Thomas Geiser, Department of Respiratory Medicine, University Hospital Bern, Switzerland; Christophe von Garnier, University Hospital Bern, Switzerland; Florian Singer, University Children's Hospital, Zurich, Switzerland; Jorgen Vestbo, Respiratory Research Group, University of Manchester, UK; Nadja Vissing, Danish Pediatric Asthma Center, University of Copenhagen, Denmark; Giorgio Pennazza, University of Rome 'Tor Vergata', Rome Italy; Marco Santoninco, University of Rome 'Tor Vergata', Rome Italy; Arnaldo D'Amico, University of Rome 'Tor Vergata', Rome Italy; Per Bakke, Haukeland University Hospital, Bergen, Norway; Bart Lambrecht, University of Gent, Gent, Belgium; Scott Wagers, BioSci Consulting, Maasmechelen, Belgium; Neil Fitch, BioSci Consulting, Maasmechelen, Belgium; Kerry Dyson, CromSource, Stirling UK; Aruna T. Bansal, Acclarogen Ltd, St. John's Innovation Centre, Cambrigde, UK; Julie Corfield, Areteva R\&D, Nottingham, UK; Richard Knowles, Arachos Pharma, UK; Tim Higgenbottam, Allergy Therapeutics, West Sussex, UK; Veit Erpenbeck, Translational Medicine, Respiratory Profiling, Novartis Institutes for Biomedical Research, Basel, Switzerland; John G. Matthews, Respiratory and Allergy Diseases, Genentech, San Francisco, CA, USA; Cecile Holweg, Respiratory and Allergy Diseases, Genentech, San Francisco, CA; Lars Larsson, AstraZeneca, Mohlndal, Sweden; Ulf Nihlen, AstraZeneca; Molndal, Sweden; Maria Gerhardsson de Verdier, AstraZeneca; Molndal, Sweden; Jeannette Bigler, Amgen Inc.; Michael J. Boedigheimer, Amgen Inc.; Wen Yu, Amgen Inc.; Xugang Hu, Amgen Inc.; Ana R. Sousa, Respiratory Therapeutic Unit, GSK, UK; Chris Compton, Respiratory Therapeutic Unit, GSK, UK David Myles, Respiratory Therapeutic Unit, GSK, UK; Elizabeth Yeyasingham, UK Clinical Operations, GSK, Stockley Park, UK; Frédéric Baribaud, Janssen R\&D, USA; Matthew Loza, Janssen R\&D, USA; Navin Rao, Janssen R\&D, USA; Anthony Rowe, Janssen R\&D, UK; Jorge Beleta, Almirall, Spain; Neil Gozzard, USB, UK; Bob Thornton, MSD, USA, Leon Carayannopoulos, MSD, USA; Klaus Fichtner, Boehringer Ingelheim Pharma GmbH \& Co. KG; Katja Nething, Boehringer Ingelheim Pharma GmbH \& Co. KG; Wolfgang Seibold, Boehringer Ingelheim Pharma GmbH, Germany; Damijan Erzen, Boehringer Ingelheim Pharma GmbH \& Co. KG; Ralf Sigmund, Boehringer Ingelheim Pharma GmbH \& Co. KG; Martina Gahlemann, Boehringer Ingelheim (Schweiz) GmbH; Kathrin Riemann, Boehringer Ingelheim Pharma GmbH \& Co. KG; Alix Berton, Boehringer Ingelheim Pharma GmbH \& Co. KG; Matthias Klüglich, Boehringer Ingelheim Pharma GmbH \& Co. KG; Eric Bucchioni, Chiesi Pharmaceuticals, SPA, Parma, Italy; Anton Vink, Philips Research Laboratories, Eindhoven, The Netherlands; Hugo Knobel, Philips Research Laboratories, Eindhoven, The Netherlands; Kjell Alving, Aerocrine AB, Stockholm, Sweden; Philip Monk, Synairgen Research Ltd, Southampton, UK; John Haughney, International Primary Care Respiratory Group, Aberdeen, Scotland; Pippa Powel, European Lung Foundation, Brussels, Belgium; Sarah Masefield, European Lung Foundation, Brussels, Belgium; Antonio Pacino, Lega Italiano Anti Fumo, Catania, Italy; Jenny Versnel, Asthma UK, London, UK; Val Hudson, Asthma UK, London, UK; David Supple, Asthma UK, UK; Breda Flood, Asthma UK, UK; Amanda Roberts, Asthma UK, UK; Michael Rutgers, Longfonds, Amersfoort, The Netherlands; Juliette Kamphuis, Longfonds, Amersfoort, The Netherlands; Pim de Boer, Longfonds,
Amersfoort, The Netherlands; Susanna Palkonen, European Federation of Allergy and Airways Diseases Patient's Associations, Brussels, Belgium.

Contributors RJBL, PJS, HKR and GtR contributed to the study protocol. RJBL, PJH, EHT, JBS-S, WJJA, TRJS, KFC, ARS, PJS, JKS and GtR contributed to the data acquisition of the ACCURATE trial; KFC, ARS and PJS contributed to the protocol and data acquisition of the U-BIOPRED study. RJBL and GtR analysed the data, PJS and HKR assisted on the interpretation. RJBL, PJS, HKR and GtR wrote the first draft of the manuscript. All authors critically revised the manuscript for intellectual content and approved the final version. RJBL and GtR had full access to all of the data in the study and take responsibility for the integrity of the data and the accuracy of the data analysis: RJBL and GtR act as guarantors.

Funding The ACCURATE study was funded by the Netherlands Organization for Health Research and Development (ZON-MW, sub-programme Effects \& Costs 80-82310-98-8627) and the Netherlands Asthma Foundation (NAF 3.4.07.044) and received non-financial support from Aerocrine. U-BIOPRED received a public-private grant by the Innovative Medicines Initiative shared by the European Union and the European Federation of Pharmaceutical Industries and Associations (grant agreement no. 115010).

Competing interests JBS-S holds stock in Grace Bros and has received consultancy fees from AstraZeneca, GlaxoSmithKline and Novartis, as well as grant funding from ACME Pharmaceutical. The institute of PJS has received a publicprivate grant by the Innovative Medicines Initiative covered by the European Union and the European Federation of Pharmaceutical Industries and Associations. KFC has received honoraria for participating in Advisory Board meetings regarding treatments for asthma and COPD for GSK, AZ, Novartis and J\&J and has received grant funding through his institution from Pfizer, GSK and Merck. ARS is an employee and holds stock in GlaxoSmithKline. HKR has participated on advisory boards for AstraZeneca, Boehringer Ingelheim, GlaxoSmithKline and Novartis, is participating on a joint data monitoring committee for AstraZeneca, GlaxoSmithKline, Merck and Novartis, has provided consultancy services for AstraZeneca, has provided continuing medical education presentations at symposia funded by AstraZeneca, Boehringer Ingelheim, GlaxoSmithKline, Novartis and Teva and has received unconditional research funding from AstraZeneca and GlaxoSmithKline. HKR is Chair of the GINA Science Committee. JKS has received research grants from Boehringer Ingelheim, GlaxoSmithKline, Chiesi and Fonds NutsOhra (1101-081), as well as non-financial support from AstraZeneca (3.4.07.044).

Ethics approval Medical Ethics Committee of the Leiden University Medical Center.

Provenance and peer review Not commissioned; externally peer reviewed.

\section{REFERENCES}

1 GINA (Global Initiative for Asthma) Global strategy for asthma management and prevention. 2015. http://www.ginasthma.org

2 Jackson DJ, Sykes A, Mallia P, et al. Asthma exacerbations: origin, effect, and prevention. J Allergy Clin Immunol 2011;128: 1165-74.

3 Ivanova Jl, Bergman R, Birnbaum HG, et al. Effect of asthma exacerbations on health care costs among asthmatic patients with moderate and severe persistent asthma. J Allergy Clin Immunol 2012;129:1229-35.

4 Loymans RJ, Gemperli A, Cohen J, et al. Comparative effectiveness of long term drug treatment strategies to prevent asthma exacerbations: network meta-analysis. BMJ 2014;:348:93009

5 Thomson NC, Chaudhuri R. Identification and management of adults with asthma prone to exacerbations: can we do better? BMC Pulm Med 2008;8:27.

6 Miller MK, Lee JH, Miller DP, et al., TENOR Study Group. Recent asthma exacerbations: a key predictor of future exacerbations. Respir Med 2007;101:481-9.

7 Kitch BT, Paltiel AD, Kuntz KM, et al. A single measure of FEV1 is associated with risk of asthma attacks in long-term follow-up. Chest 2004;126:1875-82.

8 Juniper EF, O'Byrne PM, Guyatt GH, et al. Development and validation of a questionnaire to measure asthma control. Eur Respir J 1999;14: 902-7.

9 Greenberg S. Asthma exacerbations: predisposing factors and prediction rules. Curr Opin Allergy Clin Immunol 2013;13:225-36.

10 Osborne ML, Pedula KL, O'Hollaren $\mathrm{M}$, et al. Assessing future need for acute care in adult asthmatics: the Profile of Asthma Risk Study: a prospective health maintenance organization-based study. Chest 2007;132:1151-61.

11 Eisner MD, Yegin A, Trzaskoma B. Severity of asthma score predicts clinical outcomes in patients with moderate to severe persistent asthma. Chest 2012;141:58-65.

12 Miller MK, Lee JH, Blanc PD, et al. TENOR risk score predicts healthcare in adults with severe or difficult-to-treat asthma Eur Respir J. 2006;28:1145-55.

13 Bateman ED, Buhl R, O'Byrne PM, et al. Development and validation of a novel risk score for asthma exacerbations: The risk score for exacerbations. J Allergy Clin Immunol 2015:135:1457-64.

14 Honkoop PJ, Loijmans RJ, Termeer EH, et al. Asthma Control Cost-Utility Randomized Trial Evaluation (ACCURATE) Study Group. Symptom- and fraction of 
exhaled nitric oxide-driven strategies for asthma control: A cluster-randomized trial in primary care. J Allergy Clin Immunol 2015;135:682-8.

15 Reddel HK, Taylor DR, Bateman ED, et al. An official American Thoracic Society/ European Respiratory Society statement: asthma control and exacerbations: standardizing endpoints for clinical asthma trials and clinical practice. Am J Respir Crit Care Med 2009;180:59-99.

16 White IR, Royston P, Wood AM. Multiple imputation using chained equations: issues and quidance for practice. Stat Med 2011:30:377-99.

17 Royston P, Sauerbrei W. Multivariable Model-building: a pragmatic approach to regression analysis based on fractional polynomials for modelling continuous variables. West Sussex, UK: John Wiley and Sons, 2008.

18 Steyerberg EW, Borsboom GJ, van Houwelingen $\mathrm{HC}$, et al. Validation and updating of predictive logistic regression models: a study on sample size and shrinkage. Stat Med 2004;23:2567-86.

19 Sullivan LM, Massaro JM, D'Agostino RB Sr. Presentation of multivariate data for clinical use: the Framingham Study risk score functions. Stat Med 2004;23:1631-60.

20 Steyerberg EW, Vickers AJ, Cook NR, et al. Assessing the performance of prediction models: a framework for traditional and novel measures. Epidemiology 2010;21:128-38.
21 Shaw DE, Sousa AR, Fowler SJ, et al. Clinical and inflammatory characteristics of the European U-BIOPRED adult severe asthma cohort. Eur Respir J 2015;46:1308-21.

22 Vittinghoff E, McCulloch CE. Relaxing the rule of ten events per variable in logistic and Cox regression. Am J Epidemiol 2007;165:710-18.

23 Moore WC, Bleecker ER, Curran-Everett D, et al. Characterization of the severe asthma phenotype by the National Heart, Lung, and Blood Institute's Severe Asthma Research Program. J Allergy Clin Immunol 2007;119:405-13.

24 Van Wonderen KE, Van Der Mark LB, Mohrs J, et al. Different definitions in childhood asthma: how dependable is the dependent variable? Eur Respir J 2010;36:48-56.

25 Reddel HK, Bateman ED, Becker A, et al. A summary of the new GINA strategy: a roadmap to asthma control. Eur Respir J 2015;46:622-39.

26 Korevaar DA, Westerhof GA, Wang J, et al. Diagnostic accuracy of minimally invasive markers for detection of airway eosinophilia in asthma: a systematic review and meta-analysis. Lancet Respir Med 2015;3:290-300.

27 Yawn BP, Wollan PC, Bertram SL, et al. Asthma treatment in a population-based cohort: putting step-up and step-down treatment changes in context. Mayo Clin Proc 2007;82:414-21.

28 Diette GB, Patino CM, Merriman B, et al. Patient factors that physicians use to assign asthma treatment. Arch Intern Med 2007;167:1360-6.. 


\section{Correction: Identifying patients at risk for severe exacerbations of asthma: development and external validation of a multivariable prediction model}

Loymans RJ, Honkoop PJ, Termeer EH, et al. Identifying patients at risk for severe exacerbations of asthma: development and external validation of a multivariable prediction model. Thorax 2016;71:838-46. doi: 10.1136/thoraxjnl-2015-208138

Data on the external validation are incorrect; corrections in bold.

Page 838 (Abstract): External validation yielded AUROCs of 0.69 (95\% CI 0.63 to 0.75 ; 0.63 to 0.75 and 0.63 to 0.75 ) for the three models, respectively; calibration was best for the spirometry model.

Page 840: The three models were validated externally in the Unbiased BIOmarkers in PREDiction of respiratory disease outcomes (U-BIOPRED) data set $(n=317)$, (details in online supplementary appendix I).

Page 841: U-BIOPRED participants had poor asthma symptom control and low lung function; $66.6 \%$ had a severe asthma exacerbation in the previous year (see online supplementary table A5 appendix II). The exacerbation risk was higher than in the derivation data set, with 54.9\% experiencing a severe exacerbation during 1 year follow-up. Discriminative properties of the models were lower in the external validation set (AUROC 0.69 (95\% CI 0.63 to 0.75 ), 0.69 (95\% CI 0.63 to 0.75 ) and $0.69(95 \%$ CI 0.63 to 0.75$)$ ) for the three models, respectively (figure $1 \mathrm{~B}$ ).

Page 483: the U-BIOPRED population largely consists of patients with severe asthma and this is reflected in an annual exacerbation risk of about 55\% against 13\% in the ACCURATE cohort.

Page 844: Predictive properties remained essentially intact on external validation (AUROC 0.69 ) in a population with more severe asthma and a higher (55\%) exacerbation risk.

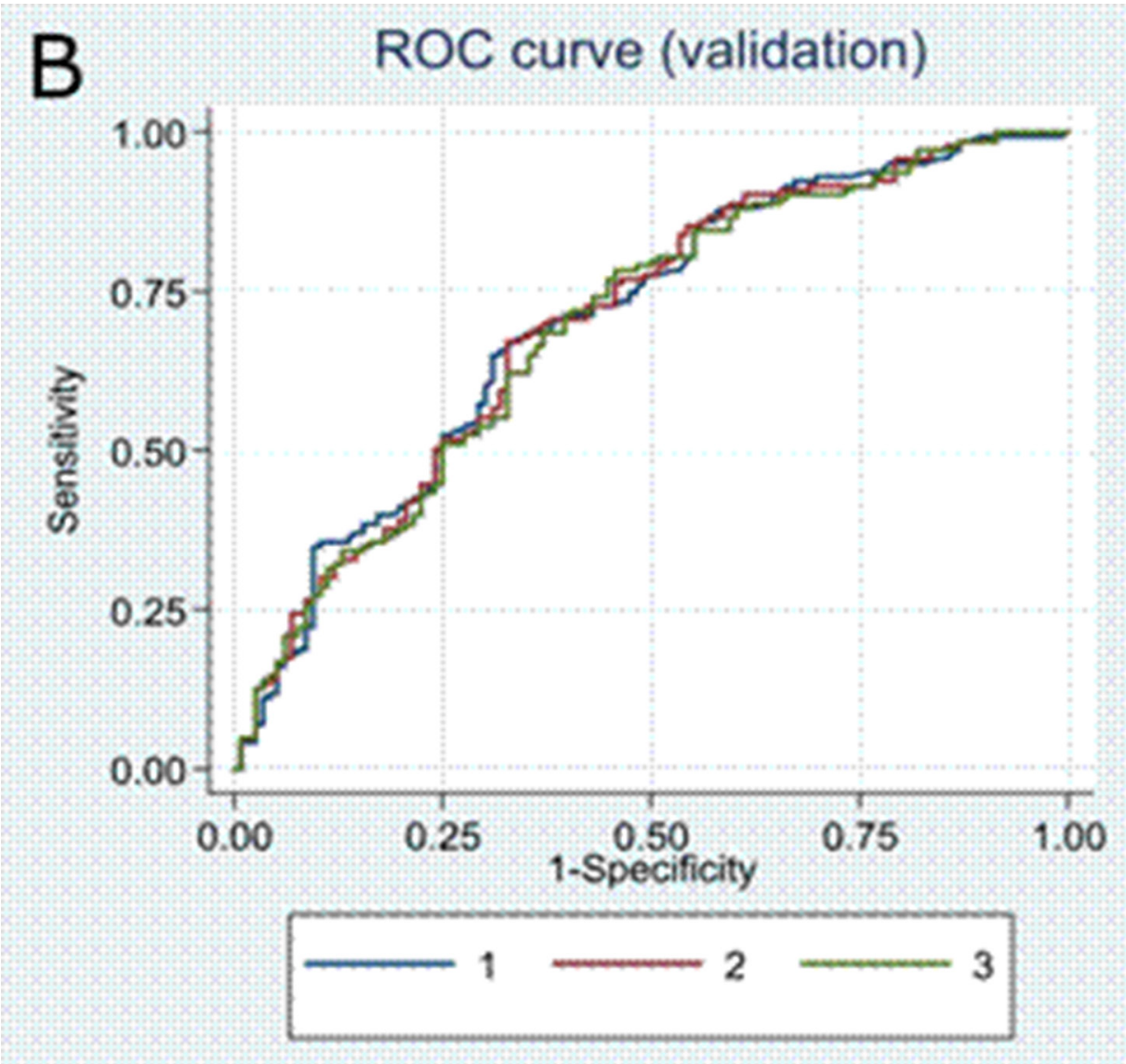



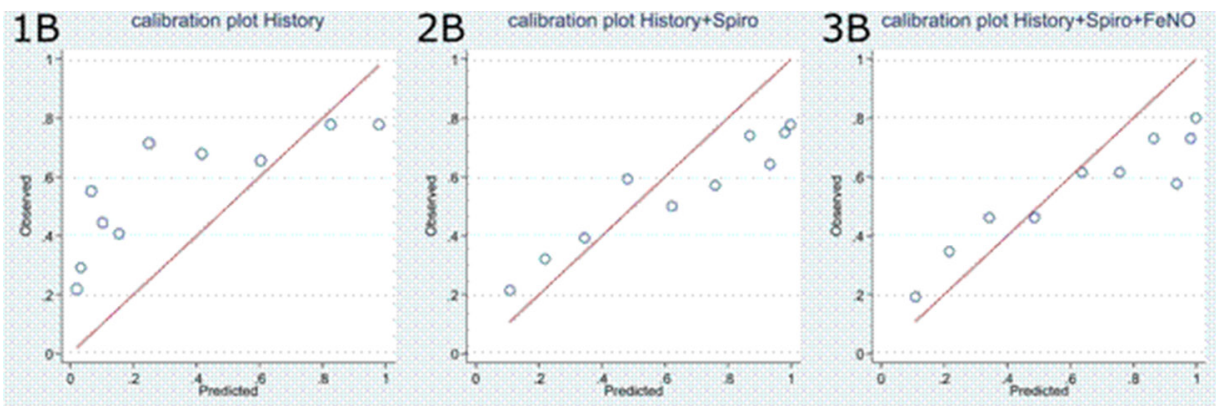

Corrected figure 2 panel 1-3B

(c) Article author(s) (or their employer(s) unless otherwise stated in the text of the article) 2018. All rights reserved. No commercial use is permitted unless otherwise expressly granted.

Thorax 2018;73:795-796. doi:10.1136/thoraxjnl-2015-208138corr1

\section{(A) Check for updates}

